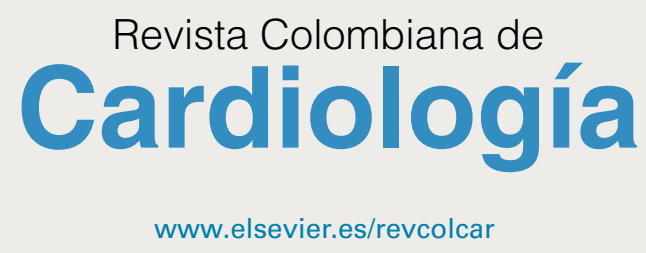

CARDIOLOGÍA DEL ADULTO - PRESENTACIÓN DE CASOS

\title{
Neuroestimulador espinal para el tratamiento de la angina cardiaca refractaria
}

\author{
Beatriz Wills $^{\mathrm{a}}$, Guillermo Monsalve ${ }^{\mathrm{b}}$, Diana Álvarez ${ }^{\mathrm{b}}$, William Amaya ${ }^{\mathrm{b}}$, \\ Jairo Moyano ${ }^{\text {b }}$ y Andrés F. Buitrago ${ }^{c, *}$
}

\author{
a Departamento de Medicina Interna, Hospital Universitario Fundación Santa Fe de Bogotá, Bogotá, Colombia \\ b Hospital Universitario, Fundación Santa Fe de Bogotá, Bogotá, Colombia \\ c Sección de Cardiología, Hospital Universitario Fundación Santa Fe de Bogotá, Bogotá, Colombia
}

Recibido el 26 de noviembre de 2014; aceptado el 2 de junio de 2015

Disponible en Internet el 29 de agosto de 2015

\section{PALABRAS CLAVE \\ Angina; \\ Dolor; \\ Tratamiento; \\ Sistema nervioso}

\section{KEYWORDS}

Angina;

Pain;

Treatment;

Nervous system

\begin{abstract}
Resumen La angina refractaria es una condición clínica frecuente y debilitante en la que los síntomas persisten a pesar de la terapia médica óptima, cuando la intervención coronaria percutánea o la revascularización miocárdica es inviable o sus riesgos injustificados. La supervivencia de los pacientes con este diagnóstico es similar a la descrita para enfermedad isquémica coronaria crónica. Las opciones terapéuticas deben enfocarse en el alivio de la angina y en la mejoría de la calidad de vida. Recientemente se han propuesto varias alternativas para el tratamiento de la angina refractaria. Se reporta la experiencia de un grupo multidisciplinario en Colombia referente al implante de un neuroestimulador medular para el manejo de la angina refractaria.

(c) 2015 Sociedad Colombiana de Cardiología y Cirugía Cardiovascular. Publicado por Elsevier España, S.L.U. Este es un artículo Open Access bajo la licencia CC BY-NC-ND (http://creativecommons.org/licenses/by-nc-nd/4.0/).
\end{abstract}

\section{Spinal neurostimulator for the treatment of refractory cardiac angina}

Abstract Refractory angina (RA) is a common and debilitating clinical condition, characterized by the persistence of symptoms despite optimal medical therapy, along with unfeasible percutaneous coronary interventions or myocardial revascularization. The survival of patients with this diagnosis is similar to that of patients with chronic ischemic heart disease. Therapeutic options should focus on relieving angina and improving quality of life. Recently, several alternatives have been proposed for the treatment of RA. We describe the experience led by

\footnotetext{
* Autor para correspondencia.

Correo electrónico: abuitrag@uniandes.edu.co (A.F. Buitrago).
} 
a Colombian multidisciplinary group, regarding the placement of a spinal neuro-stimulator for the management of a patient with RA.

(c) 2015 Sociedad Colombiana de Cardiología y Cirugía Cardiovascular. Published by Elsevier España, S.L.U. This is an open access article under the CC BY-NC-ND license (http://creativecommons.org/licenses/by-nc-nd/4.0/).

\section{Caso}

Paciente de género masculino, de 61 años de edad, con enfermedad coronaria difusa de tresvasos, compleja, ectásica y de flujos lentos, más múltiples comorbilidades: hipertensión arterial, artritis gotosa, gastropatía por consumo de antiinflamatorios no esteroideos, insuficiencia cardíaca isquémica estadio $C$ con función sistólica del ventrículo izquierdo limítrofe, carcinoma de tiroides, dislipidemia, accidente cerebrovascular isquémico y antecedente de tabaquismo. Consultó al servicio de urgencias en tres ocasiones por síndrome coronario agudo, en un periodo de dos años. El estudio de la enfermedad coronaria incluyó un cateterismo cardiaco (fig. 1) que mostró: coronaria derecha calcificada y tortuosa en todo su trayecto, con múltiples placas; enfermedad difusa de la arteria descendente posterior con vaso distal de pequeño calibre y arteria posteroventricular, con lesión del $75 \%$ en su origen; calcificación en el tercio proximal y medio de la descendente anterior, con lesión del $75 \%$ en el tercio medio, más enfermedad severa difusa en el tercio medio y distal; y calcificación en el tercio proximal de la arteria circunfleja, con lesión del $100 \%$ en la arteria del surco auriculoventricular. Debido a que no cumplía los criterios para cirugía cardiovascular, se optimizó el manejo médico dirigido a la enfermedad coronaria multivaso con warfarina, colchicina, losartán, carvedilol, trimetazidina, atorvastatina, espironolactona, mononitrato de isosorbida, amlodipino, trazadona y sertralina. También continuó con rehabilitación cardiaca, a la que asistía desde el momento en que se le diagnosticó enfermedad coronaria. No era candidato a ivabradina debido a bradicardia.

A pesar del manejo farmacológico y de mantener adherencia óptima a las recomendaciones médicas, continuó el deterioro de su clase funcional, e incrementó la frecuencia de episodios de angina. Se estudiaron otras causas de dolor torácico: enfermedad por reflujo gastroesofágico, patología de la reja costal y tromboembolia pulmonar; sin embargo, la característica del dolor (típico para agina), la respuesta parcial a los medicamentos antianginosos y los estudios correspondientes permitieron descartar dichas posibilidades.

Consultó nuevamente a urgencias por dolor torácico retroesternal, tipo opresivo, de intensidad 8/10, irradiado al dorso y a miembros superiores. Al ingreso se encontró en aceptables condiciones generales, alerta y sin signos de dificultad respiratoria; peso de $66 \mathrm{~kg}$ e índice de masa corporal (IMC) de $21 \mathrm{~kg} / \mathrm{m}^{2}$. La elevación de marcadores cardiacos junto con los hallazgos de electrocardiograma confirmaron infarto agudo de miocardio sin elevación del ST. Pese a que se solicitó valoración por clínica especializada en falla cardiaca y trasplante cardiaco, el paciente no estuvo de acuerdo con enrolarse en una lista de espera para trasplante. Además, debía practicarse resección quirúrgica del carcinoma tiroideo. También se realizó ergoespirometría para estratificar clase funcional y limitación para actividad física debido a isquemia: el consumo máximo de oxígeno correspondió a $12 \mathrm{ml} / \mathrm{min} / \mathrm{kg}$.

Debido a que la enfermedad coronaria severa no era susceptible de intervención quirúrgica o percutánea, un equipo interdisciplinario conformado por cardiología, neurocirugía y clínica de dolor de un hospital de cuarto nivel concluyó que, además de continuar con el manejo médico, el paciente se beneficiaría de la implantación de un neuroestimulador medular torácico por laminostomía. El procedimiento se practicó dos semanas posteriores al ingreso. Durante el intraoperatorio se realizó una prueba con generador de impulso no recargable para ajustar los parámetros estimulación; así mismo, se tomaron radiografías postoperatorias para confirmar la inserción correcta del mismo (fig. 2). Este constituyó el primer caso realizado en la institución,

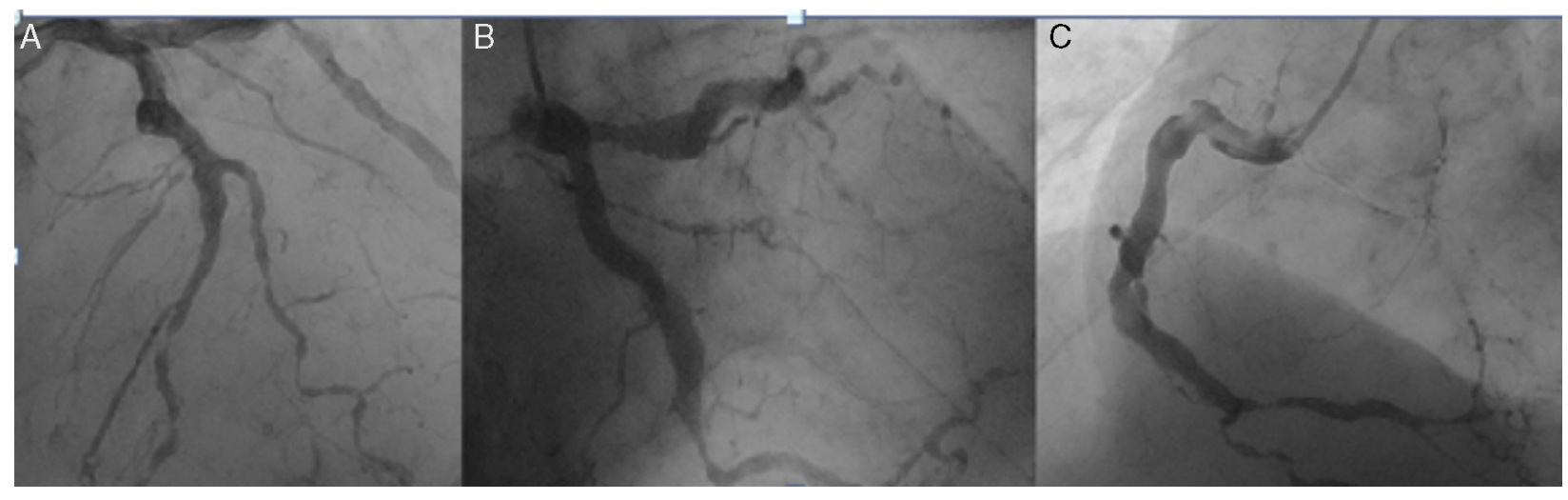

Figura 1 Cateterismo cardiaco. Enfermedad multivaso no reperfundible, ectásica y con malos lechos distales. A) Descendente anterior. B) Arteria circunfleja. C) Coronaria derecha. Ver texto. 

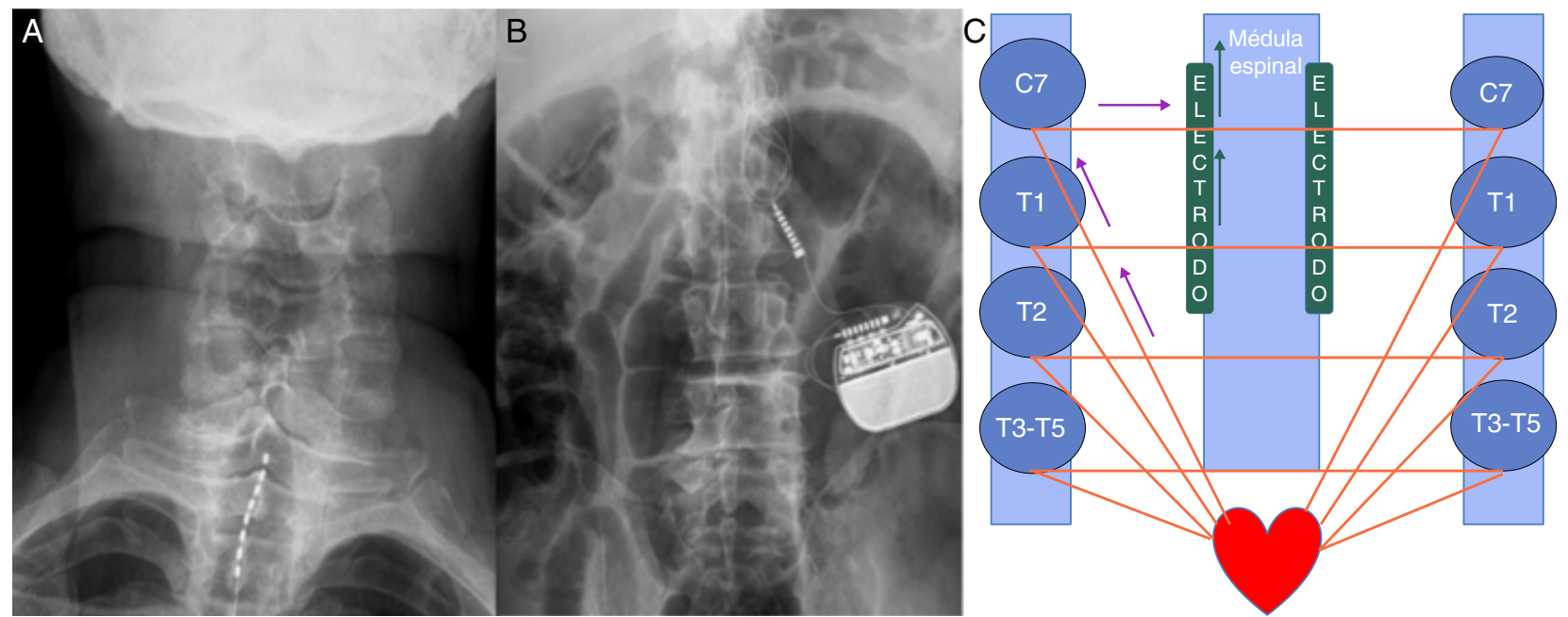

Figura 2 Radiografías postoperatorias. A) Radiografía AP de columna de unión cervicotorácica que muestra electrodo percutáneo implantado en el espacio epidural posterior en línea media a nivel de C7-T2 niveles óseos. B) Radiografía AP de abdomen simple que muestra el generador de pulsos implantado en el flanco izquierdo. C) Inervación cardiaca y efecto de la neuromodulación en los cordones posteriores de la médula espinal. Las flechas moradas indican aferencias simpáticas. Las flechas verdes indican modulación de las aferencias en los cordones posteriores de la médula espinal.

y estuvo a cargo de un neurocirujano con formación especializada en neurocirugía funcional.

De otra parte, y teniendo en cuenta el riesgo perioperatorio, se consideró como mejor opción una técnica anestésica de sedación consciente y monitorización básica convencional (presión arterial no invasiva, pulsioximetría, cánula de oxígeno con capnografía y electrocardiografía DII-V5). La estrategia farmacológica consistió en la administración de dexmedetomidina; la analgesia se logró con la administración de fentanilo y propofol para mejorar la profundidad de la sedación. Se logró una adecuada estabilidad hemodinámica al mantener un balance óptimo entre demanda y consumo miocárdico de oxígeno, sin evidencia de cambios eléctricos durante el intraoperatorio, hecho que permitió una cooperación continua en la determinación de zonas de dolor-isquemia y colaboración continua con el equipo intraoperatorio, lo cual facilitó el posicionamiento correcto del electrodo y su programación.

El paciente fue trasladado a la unidad de cuidados intensivos quirúrgicos para monitorización hemodinámica durante $24 \mathrm{~h}$; posteriormente, continuó hospitalizado durante cinco días, para ajuste de terapia anticoagulante y continuación del programa de rehabilitación cardíaca. Durante su estancia hospitalaria no refirió nuevos episodios de angina ni complicaciones postoperatorias, por lo cual se decidió dar alta hospitalaria.

Continuó en seguimiento por consulta externa de cardiología y neurocirugía funcional. En el postoperatorio de seis meses no tuvo nuevos ingresos hospitalarios, negó episodios de angina y resaltó mejoría de la clase funcional (previa ॥I/Iv, actualı/ıv). Negó disnea, edema de miembros inferiores, sincope y palpitaciones. Al examen físico se halló en buenas condiciones generales, con signos vitales adecuados. Respecto al control previo, el peso aumentó 2,5 kg.

Asiste a rehabilitación cardiaca tres veces por semana. En la última sesión alcanzó 4,1 MET, en comparación con $3 \mathrm{MET}$ previo al procedimiento.

\section{Revisión y discusión}

La Sociedad Europea de Cardiología define la angina refractaria como una condición crónica caracterizada por la persistencia del dolor o malestar torácico mayor de tres meses de duración que es resistente al manejo convencional de la enfermedad coronaria. En estos pacientes se debe demostrar que la isquemia miocárdica es la causante de los síntomas. De igual forma, los pacientes con este diagnóstico no responden a la terapia farmacológica combinada a dosis máximas -incluyendo nitratos, bloqueadores de los canales de calcio o betabloqueadores - ni a las intervenciones coronarias percutáneas, y a la revascularización miocárdica, o a ambas ${ }^{1}$.

La angina de pecho puede reflejar el estado avanzado de la enfermedad coronaria en la que el flujo de reserva coronario es insuficiente para la demanda metabólica del miocardio. Algunas características anatómicas que impiden el manejo quirúrgico son cada vez más frecuentes, por ejemplo, la presencia de enfermedad coronaria severa y difusa -como el caso que aquí se reporta-, miocardio dependiente de colaterales, múltiples reestenosis coronarias, oclusiones crónicas totales, degeneración de injerto de la vena safena, o ausencia de conductos debido a bypass coronario previo. Las comorbilidades significativas también pueden excluir la posibilidad de revascularización miocárdica en algunos pacientes. Por esto, para el equipo multidisciplinario encargado del cuidado de la angina refractaria es importante estar familiarizado con este diagnóstico y dirigir la terapia basada en los avances disponibles para su manejo ${ }^{2}$.

\section{Epidemiología}

Si bien la tasa de mortalidad anual de la angina refractaria es del $3 \%$, los pacientes sufren deterioros graves en su calidad 
de vida. En general, experimentan dolor recurrente y sostenido, hospitalizaciones frecuentes, mal estado de salud, trastornos del estado de ánimo y del sueño, limitación de las actividades diarias y pérdida de su independencia funcional. Estos desenlaces negativos tienen implicaciones considerables en cuanto a salud pública, ya que la angina refractaria puede conducir a un aumento importante de los años perdidos por discapacidad (AVAD) ${ }^{3}$.

Los datos epidemiológicos de la angina refractaria son escasos y provienen principalmente de registros de laboratorios de cateterismo cardiaco. En la Euro Heart Survey ${ }^{4}$ de 31 países, el $21 \%$ de 5.767 pacientes fueron tratados médicamente posterior a la angiografía coronaria y el $14 \%$ no fueron elegibles para revascularización tradicional. En el estudio multicéntrico e internacional Arterial Revascularization Therapies Study (ARTS), el $81 \%$ de los pacientes tratados con intervenciones coronarias percutáneas y el $62 \%$ de los tratados con bypass coronario sufrieron angina al año de seguimiento ${ }^{5}$.

Existe controversia sobre la incidencia de la angina refractaria; se estima que en Europa es de 100.000 casos nuevos por año ${ }^{6}$. Es probable que esta condición continúe en aumento debido al progreso en las técnicas de revascularización y al incremento en la supervivencia de los pacientes que han sufrido eventos coronarios. Si bien aún falta aclarar la historia natural y los predictores de mortalidad de los pacientes con angina refractaria, una cohorte prospectiva ${ }^{7}$ de 1.200 pacientes sugirió, de forma similar a otras patologías cardiovasculares, que la edad, la diabetes, la clase de angina 3 y 4 , la falla renal crónica, la disfunción del ventrículo izquierdo y/o la falla cardiaca crónica se asocian con mortalidad al año y a los nueve años posteriores al diagnóstico de angina refractaria $(p<0,0001)$. En esta cohorte, la edad promedio fue 63,5 años; el 77,5 eran hombres y el $72,4 \%$ tenían antecedente de bypass coronario.

\section{Tratamiento de la angina refractaria}

Estos pacientes representan un grupo de alto riesgo que se beneficia de recibir una atención centrada en prevención secundaria que pretende no exclusivamente prolongar la esperanza de vida sino mejorar la calidad de esta. El dolor de origen cardiaco deteriora dramáticamente la calidad de vida del enfermo y de su familia porque su presencia se identifica como una amenaza vital inminente y constante. Por esta razón, es fundamental el tratamiento analgésico ${ }^{8}$.

Así pues, el tratamiento de la angina refractaria está basado en la evidencia de las guías de práctica clínica (fig. 3) que recomiendan un manejo integral que incluya la modificación de factores de riesgo, el uso de medicamentos antianginosos y la consideración de tratamientos alternativos como la neuroestimulación de médula espinal, la contrapulsación externa potenciada (enhanced external counterpulsation [EECP]), la estimulación nerviosa eléctrica transcutánea, la revascularización con láser o incluso la angiogénesis miocárdica con terapia de células madre o génica. Estas terapias novedosas continúan en investigación y precisan de comparación en estudios clínicos aleatorizados ${ }^{9,10}$.

\section{Neuroestimulación de médula espinal}

La neuroestimulación de médula espinal se ha utilizado durante varios años para el tratamiento de la angina refractaria. En 1984, Sandric et al. ${ }^{11}$ demostraron una reducción de los síntomas de angina. Desde entonces varios estudios clínicos observacionales y controlados han mostrado efectos significativos en cuanto a los síntomas de angina y la calidad de vida. La neuroestimulación de médula espinal es un procedimiento en el cual se implantan electrodos en el espacio epidural entre C7 y T1 para estimular las columnas dorsales de la médula espinal ${ }^{12}$. El electrodo se conecta a un generador subcutáneo implantado en la pared anterior del abdomen.

Las vías de dolor anginoso son fibras aferentes simpáticas (fig. 3) que se encuentran en los ganglios de la raíz dorsal de C8-T9, especialmente en T2-T6. Las células ganglionares ascienden a través del tracto de Lissauer y progresan principalmente por los tractos espinotalámico y medial al tálamo posterior. Muchas fibras vagales se conectan al núcleo del tracto solitario y de allí al tálamo posterior, núcleo implicado en los estímulos dolorosos. El dolor torácico y del miembro superior transcurre a través de la vía simpática, mientras que el dolor ubicado en la mandíbula y el cuello viaja por vías vagales ${ }^{8}$.

Los efectos analgésicos de la neuroestimulación de médula espinal han sido bien documentados para el tratamiento de dolor neuropático; sin embargo, los mecanismos que explican los efectos antianginosos no se han establecido plenamente. Se proponen distintas teorías:

- Disminución de la percepción del dolor. La neuroestimulación de médula espinal activa selectivamente las fibras aferentes sensitivas (tipo A) mediante la estimulación eléctrica regional en la columna dorsal inhibiendo la conducción presináptica de aferentes nociceptivos (fibras A y C), lo que conlleva analgesia local. Adicionalmente, dicha neuroestimulación también aumenta la liberación de neurotransmisores inhibitorios como el ácido gamma-aminobutírico (GABA) y disminuye los niveles de neurotransmisores excitatorios como el glutamato y el aspartato. Adicionalmente, se ha encontrado que incrementa la liberación de betaendorfina. Estos cambios se traducen en un efecto cardioprotector, posiblemente al disminuir las concentraciones de noradrenalina, la contractilidad miocárdica y, por ende, el consumo de oxígeno.

- Disminución del tono simpático. Hay una reducción de la variabilidad y de la frecuencia cardiaca máxima a través de su efecto en la cadena simpática intratorácica, con lo que se impide el proceso de remodelación del miocardio secundaria al estímulo simpático.

- Aumento del flujo sanguíneo cerebral en el hipotálamo. Las técnicas no invasivas como la PET y la resonancia magnética funcional han demostrado que la sustancia gris periacueductal y el tálamo están involucrados con la percepción del dolor.

- Aumento del flujo coronario. La disminución de las catecolaminas ocasiona vasodilatación de las arterias coronarias y, por ende, mejoría en la perfusión del miocardio; incluso algunos estudios han demostrado un aumento significativo en el índice cardiaco ${ }^{13}$. 


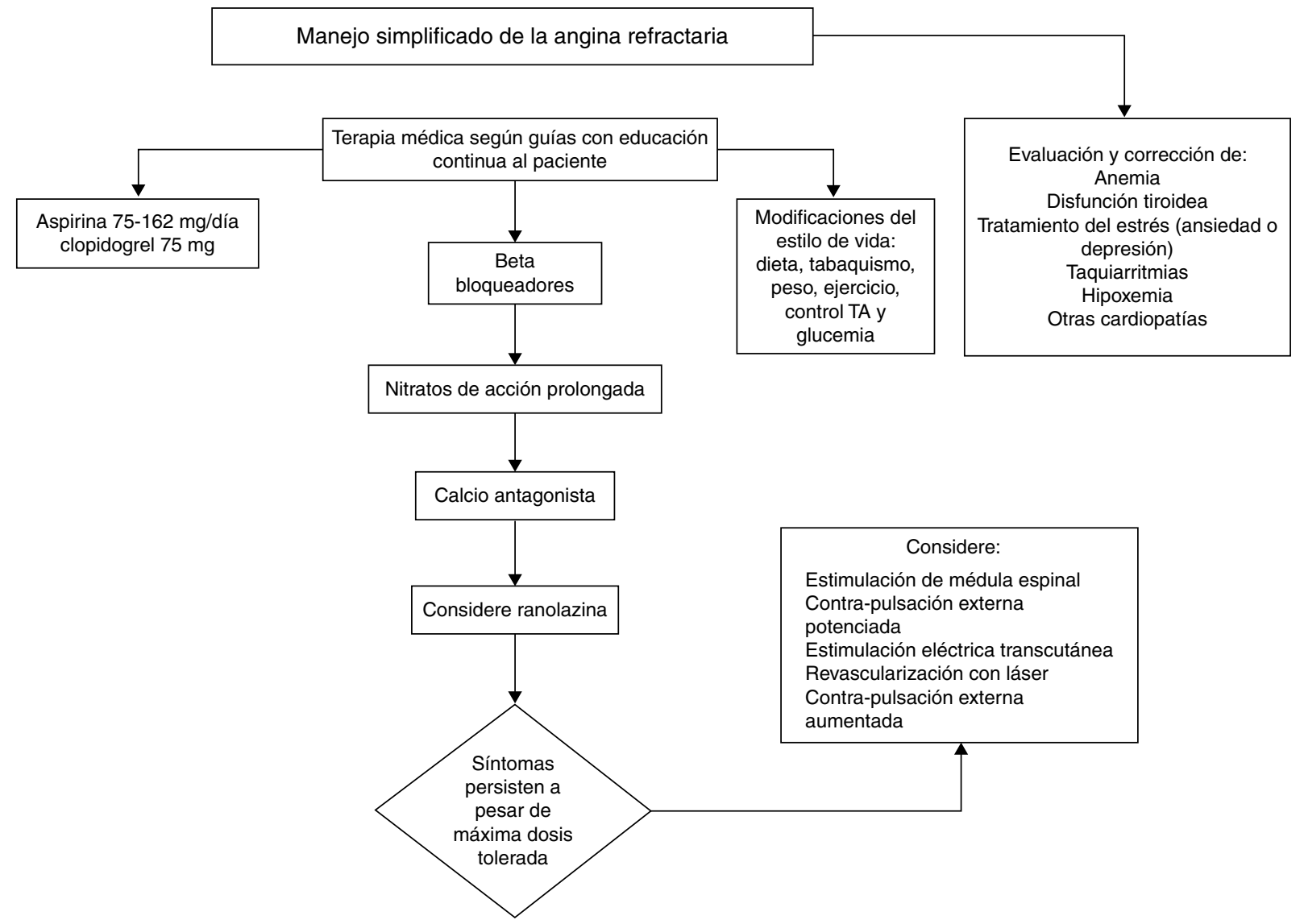

Figura 3 Manejo simplificado de angina refractaria. TA: tensión arterial. Adaptada y modificada de Mukherjee ${ }^{10}$.

Estos efectos en parte explican por qué la neuroestimulación de médula espinal reduce los signos de isquemia, incluyendo la depresión del segmento ST inducida por ejercicio y la disminución en los episodios de isquemia miocárdica transitoria. En el caso reportado, los beneficios del implante se evidenciaron temprano durante la hospitalización y se confirmaron en el seguimiento ambulatorio, pues se halló al paciente en mejor estado general, mejoró su función sexual e incluso tuvo un incremento en el índice de masa corporal. Estos hallazgos clínicos posiblemente reflejan la disminución de citoquinas proinflamatorias como la IL-1, la IL-6 y el factor de necrosis tumoral, elevadas en los pacientes con caquexia de origen cardiaco $^{13}$.

La eficacia de la neuroestimulación de médula espinal ha sido comprobada en estudios clínicos aleatorizados. TenVaarwerk et al. ${ }^{14}$ estimaron que los pacientes que recibieron este manejo tuvieron una reducción de los episodios de angina de 14 a 2,3 ataques por semana $(p<0,01)$, así como en la ingesta de nitroglicerina de 27,5 a 1,5 dosis semanales $(p<0,01)$. Documentaron, además, disminución en la frecuencia de hospitalización y en los costos asociados al cuidado coronario. Así mismo, se sabe que la neuroestimulación de médula espinal es un procedimiento seguro y reversible; los efectos adversos más comunes son migración o daño de los electrodos e infección, con una frecuencia reportada del 15 y del $5 \%$, respectivamente ${ }^{14}$.
Es, igualmente, una alternativa viable con una relación costo-beneficio favorable, y aún faltan estudios que demuestren superioridad frente a otras alternativas. El procedimiento y los equipos con registro INVIMA tuvieron un costo aproximado de 50 millones de pesos colombianos. En Colombia, este es un procedimiento que aún no está incluido en el plan obligatorio de salud; sin embargo, fue aprobado por el seguro médico del paciente (específicamente por la empresa promotora de salud).

En la actualidad el Colegio Americano de Cardiología y la Sociedad Europea de Cardiología clasifican el nivel de evidencia de esta terapia como grado ıb, y ambos consideran la neuroestimulación de médula espinal para mejorar los síntomas y la calidad de vida de los pacientes con angina refractaria incapacitante y sin posibilidad para revascularización miocárdica ${ }^{15}$.

Por último, es importante aclarar que la neuroestimulación de médula espinal no es una contraindicación para el trasplante cardiaco, e incluso puede ser un puente para un manejo definitivo. Sin embargo, en este caso no se realizó inicialmente por voluntad del paciente.

\section{Conclusiones}

Se reporta un caso exitoso en Colombia de neuroestimulación de médula espinal para el manejo de la angina 
refractaria. Si bien este procedimiento no reemplaza la revascularización miocárdica, es una alternativa viable que tiene como prioridad mejorar la calidad de vida. La terapia de la angina refractaria debe acompañarse de una estrategia multidisciplinaria conformada por cardiología clínica e intervencionista, clínica de dolor, neurocirugía funcional, psicología y rehabilitación física. La elección de la terapia para angina refractaria debe ser individualizada, teniendo en cuenta la disponibilidad, la experiencia clínica, los costos $\mathrm{y}$, en particular, los riesgos del tratamiento.

\section{Responsabilidades éticas}

Protección de personas y animales. Los autores declaran que para esta investigación no se han realizado experimentos en seres humanos ni en animales.

Confidencialidad de los datos. Los autores declaran que han seguido los protocolos de su centro de trabajo sobre la publicación de datos de pacientes.

Derecho a la privacidad y consentimiento informado. Los autores declaran que en este artículo no aparecen datos de pacientes.

\section{Conflicto de intereses}

Los autores declaran no tener conflicto de intereses.

\section{Agradecimientos}

A los Doctores Carlos Andrés Carvajal y Carlos Guerrero.

\section{Bibliografía}

1. Fox K, García M, Ardissino D, Buszman P, Camici P, Crea F, et al. ESC Committee for Practice Guidelines (CPG). Guidelines on the management of stable angina pectoris: Executive summary. The Task Force on the Management of Stable Angina Pectoris of the European Society of Cardiology. Eur Heart J. 2006;27:1341-81.

2. McGillion M, Arthur HM, Cook A, Carroll SL, Victor JC, L'Allier PL, et al. Management of patients with refractory angina: Canadian Cardiovascular Society/Canadian Pain Society joint guidelines. Can J Cardiol. 2012;28:S20-41.
3. Angina R. The problem of chronic refractory angina. Eur Heart J. 2002;23:355-70.

4. Lenzen M, Boersma E, Bertrand M, Maier W, Moris C, Piscione $F$, et al. Management and outcome of patients with established coronary artery disease: The Euro Heart Survey on coronary revascularization. Eur Heart J. 2005;26:1169-79.

5. Serruys PW, Unger F, Sousa JE, Jatene A, Bonnier HJ, Schönberger JP, et al. Comparison of coronary-artery bypass surgery and stenting for the treatment of multivessel disease. N Eng J Med. 2001;344:1117-24.

6. Taylor RS, De Vries J, Buchser E, DeJongste MJ. Spinal cord stimulation in the treatment of refractory angina: Systematic review and meta-analysis of randomised controlled trials. BMC Cardiovasc Disord. 2009;9:13.

7. Henry TD, Satran D, Hodges JS, Johnson RK, Poulose AK, Campbell $A R$, et al. Long-term survival in patients with refractory angina. Eur Heart J. 2013;34:2683-8.

8. De Vries J, Foreman RD, DeJongste MJ. The anti-ischemic effects of electrical neurostimulation in the heart. Cleveland Clin J Med. 2007;74 Suppl 1:S42.

9. Arora RR, Chou TM, Jain D, Fleishman B, Crawford L, McKiernan $T$, et al. Effects of enhanced external counterpulsation on Health Related Quality of Life continue 12 months after treatment: A substudy of the Multicenter Study of Enhanced External Counterpulsation. J Invest Med. 2002;50:25-32.

10. Mukherjee D. Management of refractory angina in the contemporary era. Eur Heart J. 2013;34:2655-7.

11. Sandric S, Meglio M, Bellocci F, Montenero A, Scabbia E, D'Annunzio V. Clinical and Electrocardiographic Improvement of Ischemic Heart Disease after Spinal Cord Stimulation. Advances in Stereotactic and Functional Neurosurgery 6. Springer; 1984. p. 543-6.

12. Song JJ, Popescu A, Bell RL. Present and potential use of spinal cord stimulation to control chronic pain. Pain Physician. 2014; 17:235-46.

13. Kosmala W, Derzhko R, Przewlocka-Kosmala M, Orda A, Mazurek W. Plasma levels of TNF- $\alpha, \mathrm{IL}-6$, and IL-10 and their relationship with left ventricular diastolic function in patients with stable angina pectoris and preserved left ventricular systolic performance. Coronary Artery Dis. 2008;19:375-82.

14. TenVaarwerk I, Jessurun G, DeJongste M, Andersen C, Mannheimer C, Eliasson T, et al. Clinical outcome of patients treated with spinal cord stimulation for therapeutically refractory angina pectoris. Heart. 1999;82:82-8.

15. Montalescot G, Sechtem U, Achenbach S, Andreotti F, Arden C, Budaj A, et al. 2013 ESC guidelines on the management of stable coronary artery disease: The Task Force on the management of stable coronary artery disease of the European Society of Cardiology. Eur Heart J. 2013;34:2949-3003. 